\title{
Immunohistochemical localisation of protein tyrosine kinase receptors Tie- 1 and Tie-2 in synovial tissue of rheumatoid arthritis: correlation with angiogenesis and synovial proliferation
}

\author{
Takeshi Uchida, Masahiro Nakashima, Yashuhiro Hirota, Yoichi Miyazaki,
} Tomoo Tsukazaki, Hiroyuki Shindo

\begin{abstract}
Objective-To investigate the involvement of Tie-1 and Tie-2, receptor tyrosine kinases required for angiogenesis, in synovial proliferation and angiogenesis of rheumatoid arthritis (RA).

Methods-Synovial tissues from 10 patients with RA and three control subjects were analysed by double immunohistochemistry and reverse transcriptase polymerase chain reaction (RT-PCR).

Results-Expression of Tie-1 and Tie-2 was seen in all synovia, but predominantly in papillary projected portions. In synovial lining cells, Tie-2 was expressed mainly in the basal layer and frequently colocalised with vimentin and proliferating cell nuclear antigen (PCNA), whereas Tie-1 was also expressed in the superficial layer. In stromal cells, Tie-2 immunoreactivity was restricted to vimentin positive fibroblast-but not macrophage derived cells, whereas Tie-1 expression was not dependent on the phenotype. Tie receptors were also highly expressed in the endothelium and surrounding pericytes of capillaries scattered over the papillary proliferated synovium without notable difference in the expression of the two receptors. Furthermore, Tie positive vessels often overexpressed PCNA. In normal synovia, expression of Tie receptors was restricted to the capillary endothelium. RT-PCR confirmed the expression of Tie-1 and Tie-2 in RA synovial tissues and also in the cultured synoviocytes.

Conclusion-The results suggest the possible involvement of overexpressed Tie-1 and Tie-2 in synovial lining and stromal cells in the pathophysiology of RA synovitis, probably through distinct mechanisms. Furthermore, expression of Tie receptors in actively growing vasculature may reflect the direct involvement of these receptors in angiogenesis and subsequent vascularisation.

(Ann Rheum Dis 2000;59:607-614)
\end{abstract}

In addition to synovial proliferation, angiogenesis is a major pathological feature of rheumatoid arthritis (RA). Blood vessel growth and involution are markedly increased in RA synovium. It is well known that blood vessels in actively proliferating synovia are constantly remodelling, and several proliferation markers are expressed in many dividing endothelia. ${ }^{1}{ }^{2}$ Furthermore, several angiogenic factors, such as vascular endothelial growth factor, fibroblast growth factor, and soluble E-selectin, are overexpressed in inflamed joints..$^{3-5}$ Based on this evidence, angiogenesis is currently considered as a candidate target in the treatment of RA. Inhibition of blood vessel growth might be beneficial by attenuating synovitis. In fact, several anti-angiogenic agents of chemical or synthesised peptides have been used experimentally in animal models of arthritis, and shown to inhibit joint inflammation with concomitant decrease of synovial proliferation. ${ }^{67}$

Tie- 1 and Tie- 2 are endothelial cell-specific tyrosine kinase receptors expressed in the vascular system from the early stages of embryogenesis. ${ }^{8}{ }^{9}$ These receptors have unique extracellular domains that are not seen in other tyrosine kinase receptors, and overall identity of the predicted amino acid sequences between these receptors is around $50 \%$. Recent target disruption studies have shown that Tie receptors have a crucial role in angiogenesis. Dysfunction of either Tie- 1 or Tie- 2 causes a defect in capillary network formation, resulting in oedema and haemorrhage. ${ }^{10}{ }^{11}$ More recently, angiopoietin- 1 and -2 were isolated as ligands for Tie-2, though Tie-1 is still an orphan receptor. ${ }^{12}{ }^{13}$ Angiopoietin-1 induces autophosphorylation of Tie-2, and stimulates proliferation and maturation of smooth muscle cells surrounding blood vessels. In knockout mice of angiopoietin-1, proliferation of the endothelium and capillary formation may take place, but most mice die at the embryonic stage owing to reciprocal interactions between endothelial cell and surrounding matrix or mesenchymal cells. ${ }^{14}$ In contrast, angiopoietin-2 acts as an antagonist by competitive binding with angiopoietin-1 through Tie-2. ${ }^{13}$ However, it seems that angiopoietin-2 is also required to initiate neovascularisation. ${ }^{15}$ These findings strongly implicate the functional requirement of the angiopoietin-Tie system in angiogenesis and subsequent microvascular maintenance.

Using immunohistochemistry, we show here that Tie-1 and Tie-2 are expressed not only in vessel walls but also in synovial lining and stromal cells, and that the expression of these receptors is associated with the degree of vascularity and synovial proliferation. Bearing in mind these findings, we discuss the possible functional involvement of Tie receptors in angiogenesis and proliferation of RA synovium.
Accepted for publication 9 February 2000 
Table 1 Patients'characteristics

\begin{tabular}{lllllll}
\hline Patient No & Sex & Age (years) & $\begin{array}{l}\text { Disease duration } \\
\text { (years) }\end{array}$ & Affected joint & Drug & Stage \\
\hline 1 & F & 55 & 22 & Hip & P, D & IV \\
2 & F & 68 & 5 & Hip & P & IV \\
3 & F & 57 & 22 & Ankle & P & IV \\
4 & F & 49 & 8 & Hip & P, D & III \\
5 & F & 63 & 11 & Hip & P & III \\
6 & F & 75 & 7 & Knee & P & III \\
7 & F & 79 & 5 & Knee & P & III \\
8 & F & 69 & 8 & Knee & P & IV \\
9 & F & 22 & 7 & Klbow & P & IV \\
10 & M & 78 & 7 & Hip & - & II \\
$11^{\star}$ & F & 69 & - & Hip & - & - \\
$12^{\star}$ & F & 85 & - & Hip & - & - \\
$13^{\star}$ & F & 72 & - & & & \\
\hline
\end{tabular}

$\mathrm{P}=$ prednisolone; $\mathrm{D}=$ disease modifying antirheumatic drugs.

^Patients 11-13 are subjects with femoral neck fracture.

\section{Materials and methods}

TISSUE SAMPLES

Ten synovial tissue samples were obtained at the time of joint replacement surgery or synovectomy from patients with RA after informed consent. As normal controls, three joint capsules were also obtained during femoral head replacement surgery from patients with femoral neck fracture. Table 1 summarises the clinical information on each patient. The diagnosis of RA was based on the criteria of the American College of Rheumatology. ${ }^{16}$ Each sample was fixed in $10 \%$ formalin immediately after removal. For reverse transcriptase polymerase chain reaction (RT-PCR), tissues were cryopreserved at $-80^{\circ} \mathrm{C}$.

IMMUNOHISTOCHEMISTRY

Table 2 lists the primary antibodies used in this study. Formalin fixed and paraffin embedded tissues were used for the immunohistochemistry of Tie-1 and Tie-2. Immunostaining was performed using the avidin-biotin complex technique as described previously. ${ }^{17}$ Paraffin embedded tissues were cut into $2 \mu \mathrm{m}$ thick sections, deparaffinised in xylene, and rehydrated in phosphate buffered saline. After immersion in $0.3 \% \mathrm{H}_{2} \mathrm{O}_{2}$ to block endogenous peroxidase activity, sections were preincubated with $10 \%$ normal goat serum to prevent non-specific binding and then incubated overnight at $4^{\circ} \mathrm{C}$ with anti-Tie-1 or anti-Tie-2-specific polyclonal antisera. The slides were subsequently incubated with biotinylated goat antirabbit IgG antibody for one hour, followed by avidinperoxidase for 30 minutes, and coloured with 3-amino-9-ethylcarbazole hydrochloride. Control experiments included incubation with non-immunised rabbit serum instead of the primary antibody and an immunoabsorption test by each antigen (SC-342 P and SC-324 P for Tie-1 and Tie-2, respectively); they did not show any staining.

To determine the phenotype of cells immunoreactive to Tie- 1 and Tie- 2 , double immunohistochemistry of Tie-1/Tie-2 and CD68, vimentin, CD34, $\alpha$ smooth muscle actin ( $\alpha$ SMA) was performed as described previously. ${ }^{18}$ In addition, in an attempt to show proliferating cells in the synovium, double staining with proliferating cell nuclear antigen (PCNA) was performed. The primary antibody for the first immunohistochemical reac-
Table 2 Antibodies used in this study

\begin{tabular}{llll}
\hline Antibody & Origin & Supplier & Dilution \\
\hline Tie-1 & Rabbit & Santa-Cruz & $1 / 200$ \\
Tie-2 & Rabbit & Santa-Cruz & $1 / 500$ \\
CD68 & Mouse & Zymed & Prediluted \\
Vimentin & Mouse & Dako & Prediluted \\
CD34 & Mouse & Novocastra & $1 / 25$ \\
$\alpha$ SMA $^{\star}$ & Mouse & Enzo Diagnostics & $1 / 150$ \\
PCNA $^{\star}$ & Mouse & Dako & $1 / 200$ \\
\hline${ }^{\star} \alpha$ SMA $=\alpha$ smooth muscle actin; PCNA = proliferating cell \\
nuclear antigen.
\end{tabular}

tion was Tie-1 or Tie-2, followed by secondary staining with mouse antisera. The second immunohistochemical reactions for CD68, vimentin, CD34, aSMA, and PCNA were visualised using alkaline phosphatase conjugated antimouse IgG antibody with a mixture of 5-bromo-4-chloro-3-indolyl phosphate and nitroblue tetrazolium chloride colouration. Control slides in double immunohistochemistry experiments were carried out by incubation with only the secondary antibody, without added primary antibody during the second immunohistochemical procedure and did not show any staining in the second reaction.

Under $\times 100$ magnification, the staining frequencies of Tie- 1 and Tie-2 in each component were evaluated semiquantitatively by three observers, as follows: $+++=>50 \%,++=$ $20-50 \%,+=5-20 \%$, and $+/-=<5 \%$.

RT-PCR

Total RNA was extracted from each tissue sample by a modified guanidine-phenol method. After denaturing with heat incubation for 10 minutes at $70^{\circ} \mathrm{C}$, complementary DNA (cDNA) was synthesised from $1 \mu \mathrm{g}$ of RNA using Molony murine leukaemia virus reverse transcriptase for one hour at $37^{\circ} \mathrm{C}$ in the presence of oligo-dT primer. PCR was performed using specific primers designed on the 3' region of each cDNA as follows: Tie-1, sense: GCCATGATCAAGAAGGACGG, antisense: GTTCTCTCCGACCAGCACAT; Tie-2, sense: TGTTCCTGTGCCACAGGCTG, antisense: CACTGTCCCATCCGGCTTCA; G3PDH, sense: ACCACAGTCCATGCCATCAC, antisense: TCCACCACCCTGTTGCTGTA. The thermal profile was 15 seconds at $95^{\circ} \mathrm{C}, 15$ seconds at $55^{\circ} \mathrm{C}, 30$ seconds at $72^{\circ} \mathrm{C}$. The amplification cycles of Tie- 1 and Tie- 2 were both 35 . For glyceraldehyde-3-phosphate dehydrogenase (G3PDH), amplification was reduced to 25 cycles. The expected sizes of Tie-1, Tie-2, and G3PDH were 407 bp, 317 bp, and $750 \mathrm{bp}$, respectively. To exclude possible contamination of genomic DNA, PCR was also applied to reactions without RT.

RT-PCR with the same procedures and conditions was also applied to cultured fibroblastlike synoviocytes obtained from two independent patients and subcultured five times in RPMI based culture medium.

\section{Results}

EXPRESSION OF TIE-1 AND TIE-2 IN SYNOVIAL LINING AND STROMAL CELLS

Immunoreactivity for Tie-1 or Tie- 2 was seen in blood vessels as well as in synovial lining and stromal cells in all RA tissues, while only the 
A

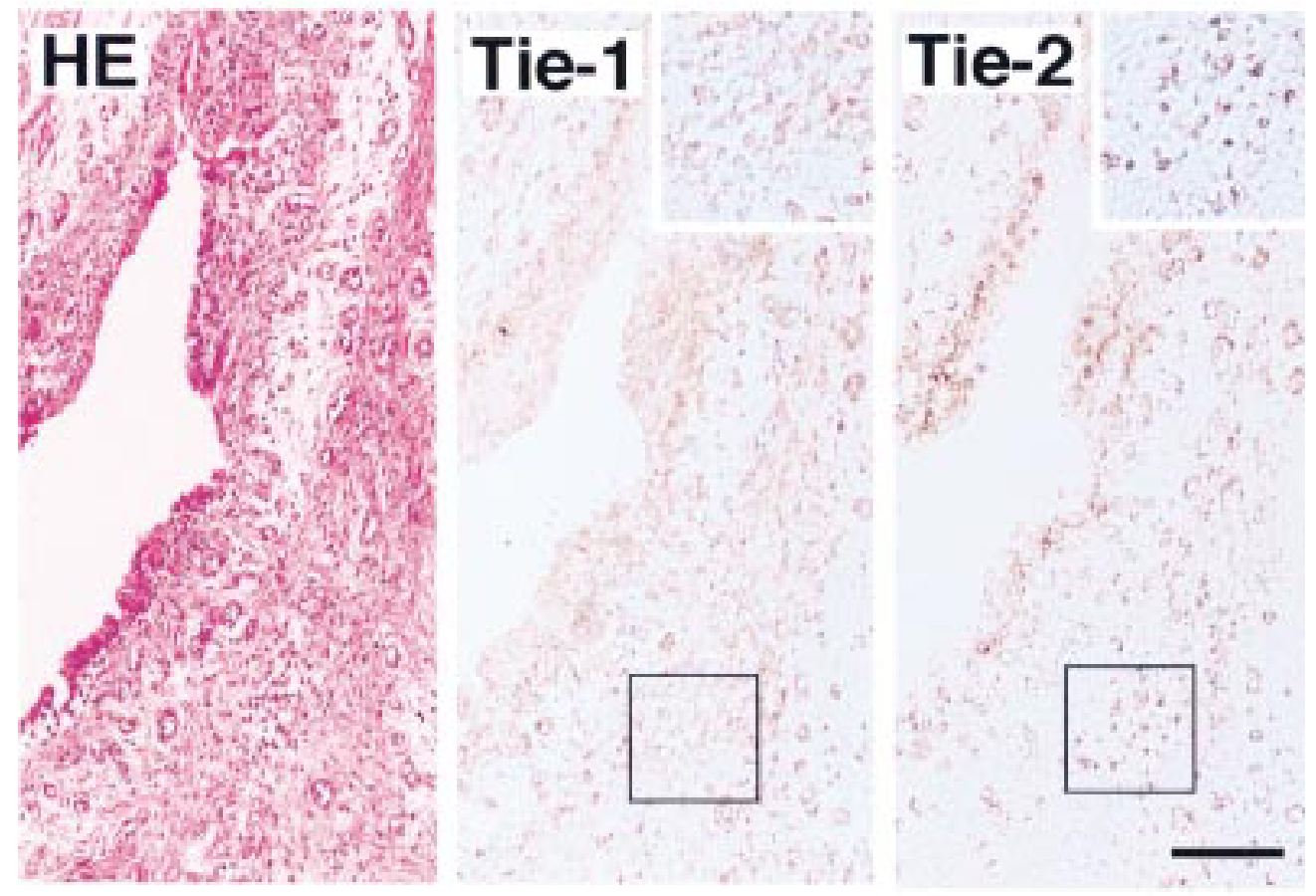

B

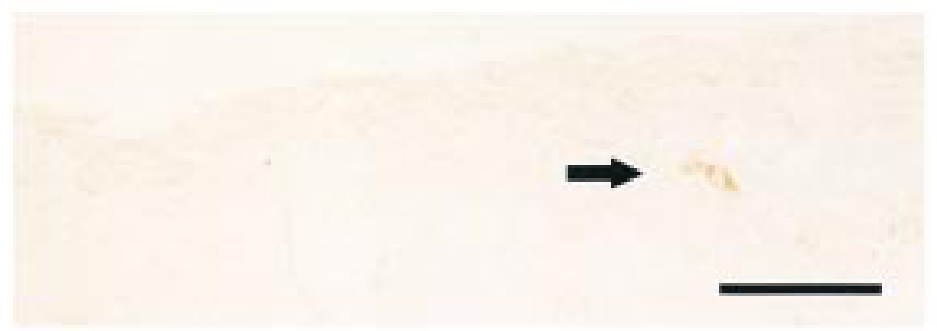

Figure 1 Expression of Tie-1 and Tie-2 in rheumatoid arthritis $(R A)(A)$ and control (B) synovia. Three serial sections from patient No 4 were used for haematoxylin and eosin (HE) staining (left panel), immunostaining for Tie-1 (middle panel), and Tie-2 (right panel). Expression of Tie-1 and Tie-2 was widely distributed in RA synovium, such as lining cells and stromal components, including capillaries. The distribution of Tie-1 immunoreactivity was more widespread than that of Tie-2. Note the restricted expression of Tie-2 to the basal layer of stratified lining cells. Although the number of Tie-2 positive cells was somewhat less than for Tie-1, the intensity of staining was stronger than Tie-1 in both lining and stromal cells except for capillaries (insets). In control patient No 11, only the capillary components (arrow) were positive for Tie-2. Bars $=100 \mu \mathrm{m}$.

capillary endothelium was stained in control subjects (figs $1 \mathrm{~A}$ and $\mathrm{B}$ ). However, the stained cell number and staining intensity were different among samples (table 3). In general, Tie receptors were preferentially expressed in the papillary projected portions containing a high density of vascular and stromal cells. Comparison of the distribution of Tie-1 and Tie-2 showed that Tie-1 tended to be expressed widely throughout stratified synovial lining cells (fig 2A), whereas Tie-2 expression was restricted to the basal layer. In addition, Tie-1 immunoreactive stromal cells were distributed more widely than those of Tie-2.

To identify the phenotype of cells expressing each Tie receptor, double staining with CD68 and vimentin antibodies, markers for histiocytic and fibroblastic cells, respectively, was performed. In synovial layers, CD68 was expressed predominantly in the superficial layer and did not colocalise with Tie-2 (fig 2B).
Table 3 Expression levels of Tie-1 and Tie-2 in the rheumatoid synovium of each patient

\begin{tabular}{|c|c|c|c|c|c|c|}
\hline \multirow{2}{*}{$\begin{array}{l}\text { Patient } \\
\text { No }\end{array}$} & \multicolumn{3}{|l|}{ Tie-1 } & \multicolumn{3}{|l|}{ Tie-2 } \\
\hline & Lining & Sublining & Vessels & Lining & Sublining & Vessels \\
\hline 1 & $+/-$ & $+/-$ & $+/-$ & $+/-$ & $+/-$ & + \\
\hline 2 & $+/-$ & $+/-$ & $+/-$ & + & + & ++ \\
\hline 3 & + & ++ & $+/-$ & $+/-$ & $+/-$ & + \\
\hline 4 & ++ & +++ & +++ & ++ & ++ & +++ \\
\hline 5 & $+1-$ & $+1-$ & $+1-$ & + & + & ++ \\
\hline 6 & + & $+/-$ & + & +++ & + & +++ \\
\hline 7 & $+1-$ & $+/-$ & $+1-$ & ++ & ++ & ++ \\
\hline 8 & $+/-$ & $+/-$ & +++ & ++ & +++ & +++ \\
\hline 9 & $+1-$ & $+/-$ & + & + & $+/-$ & ++ \\
\hline 10 & + & $+/-$ & $+/-$ & ++ & + & ++ \\
\hline
\end{tabular}

,,++++++ , and $+/-$ represent staining frequencies of $>50 \%$, $20-50 \%, 5-20 \%$, and $<5 \%$, respectively, as described in "Materials and methods". Serial sections were stained in two separate procedures, and showed the same results.

In contrast, vimentin was expressed in the deep layer and often colocalised with Tie-2 (fig 2C). A similar trend was seen in stromal cells; a large proportion of Tie- 2 positive cells co-stained 

$\mathrm{H})$, though not all vimentin positive cells expressed Tie-2. In contrast, Tie-1 positive cells co-stained with either CD68 or vimentin. with vimentin but not with CD68 (figs $2 \mathrm{G}$ and

However, there were still large numbers of singly stained cells (figs $2 \mathrm{E}$ and $\mathrm{F}$ ). To assess the correlation between Tie-2 expression and cell proliferation, double staining with PCNA was
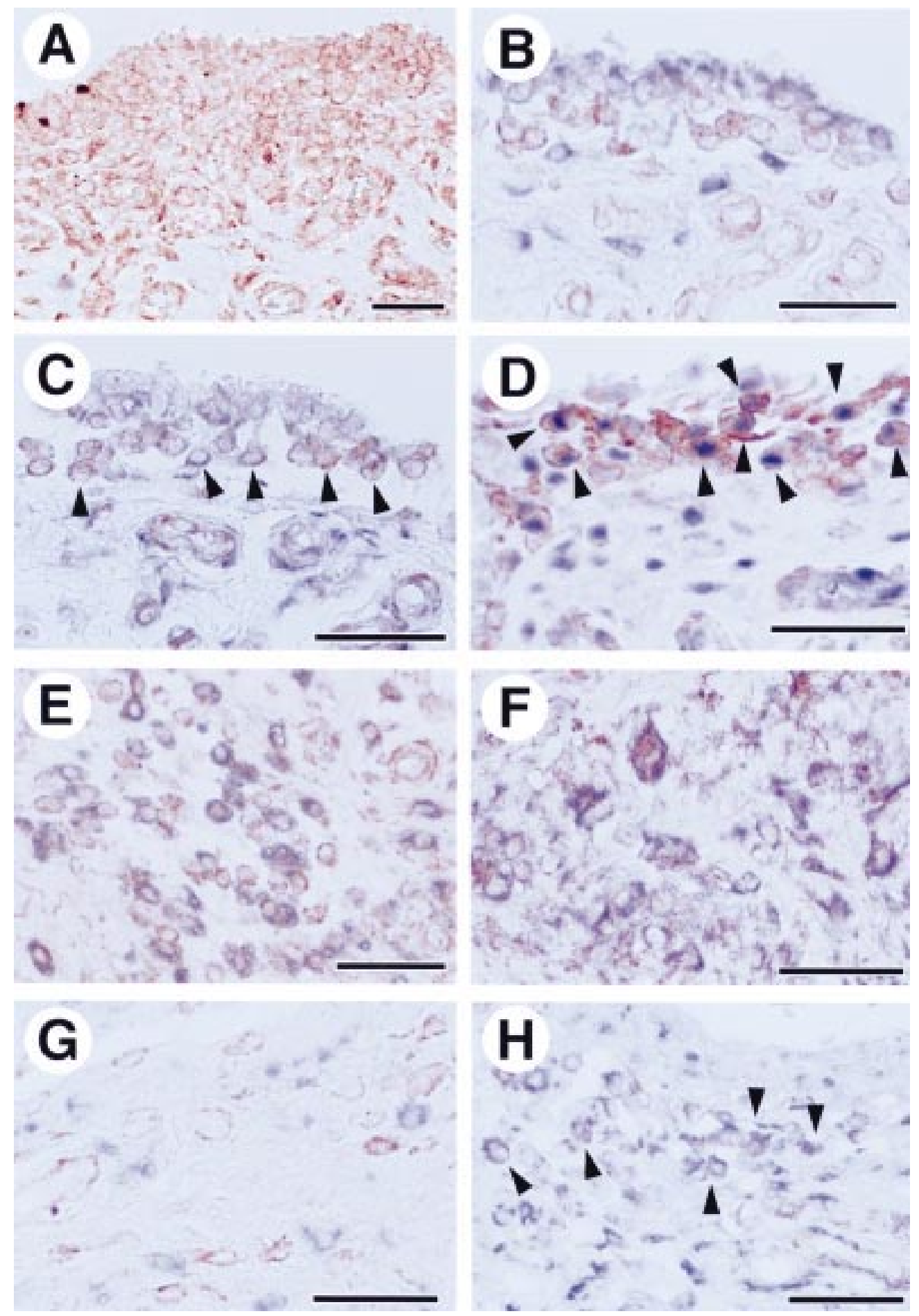

Figure 2 Double immunohistochemistry of synovial lining cells $(A-D)$ and stromal cells $(E-H)$. (A) Immunostaining for Tie-1. Tie-1 expression was seen throughout stratified synovial lining cells. (B) Double staining for Tie-2 (red) and CD68 (blue). Tie-2 immunoreactivity was predominantly localised in the basal layer of synovial lining cells, whereas CD68 immunoreactivity was restricted to the superficial layer. (C) Double staining with Tie-2 (red) and vimentin (blue). Colocalisation of Tie-2 and vimentin expression was obvious in the basal layer of the synovium (arrowheads) on the serial section shown in (B). (D) Double staining of Tie-2 (red) and proliferating cell nuclear antigen (PCNA) (blue). Similarly to section C, colocalisation of Tie-2 and PCNA was restricted to the basal layer (arrowheads). (E, F) Double staining of Tie-1 (red) and CD68 (blue)/vimentin (blue) in stromal cells. Tie-1 expression was frequently colocalised with both CD 68 (E) and vimentin $(F) .(G, H)$ Double staining of Tie-2 (red) and CD68 (blue)/vimentin (blue) in stromal cells. In contrast with Tie-1, the distribution of Tie-2 expression was completely distinct from that of $C D 68(G)$. On the other hand, Tie-2 expression was frequently concordant with vimentin positive cells $(H)$ (arrowheads). $A-D=$ patient No 4, $E-H=$ patient No 8. Bars in each panel $=50 \mu \mathrm{m}$. 
also performed. PCNA positive cells were mainly distributed in the basal layer of stratified synovial lining cells, and colocalised with Tie-2 positive cells (fig 2D). Several stromal cells also overexpressed PCNA. However, no distinct difference was noted

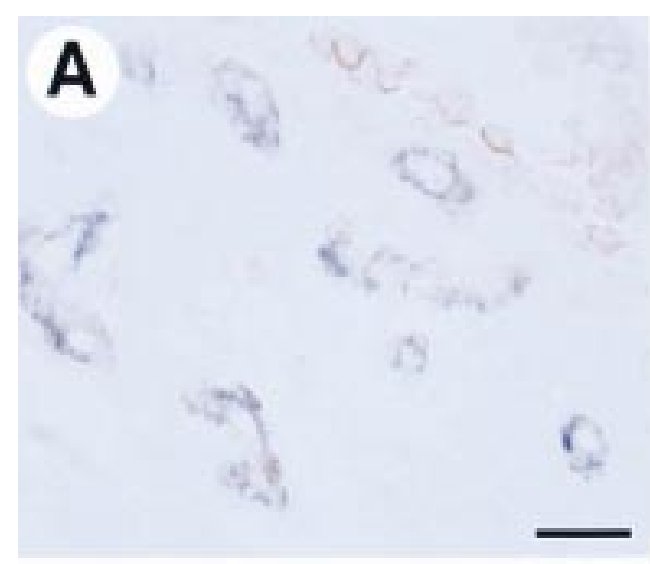

in the distribution pattern between PCNA and Tie receptors (data not shown). Comparison of the staining of Tie receptors with clinical information showed no obvious relation in the expression profiles of either Tie-1 or Tie-2.
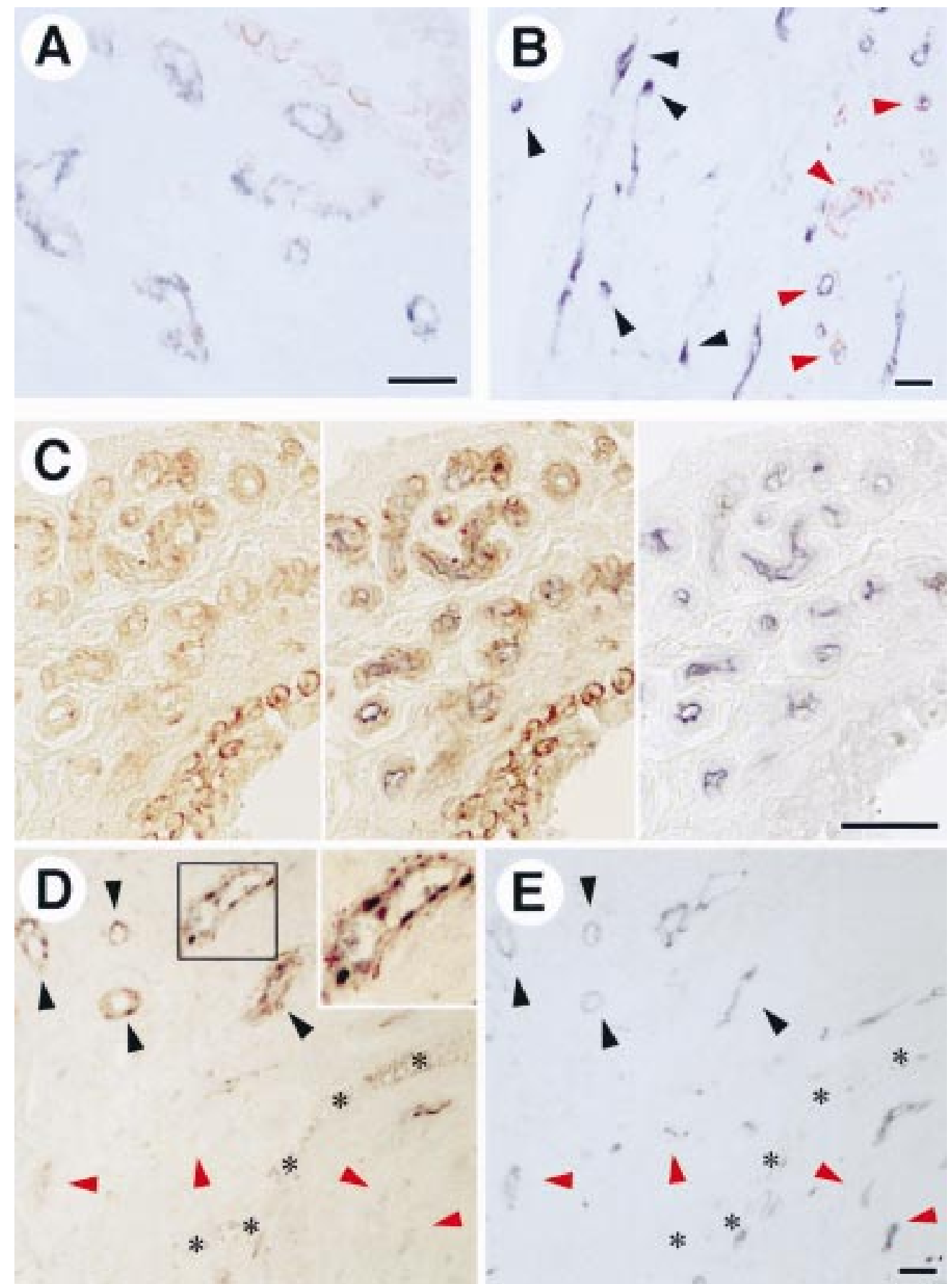

Figure 3 Tie-2 expression in the synovial vascular component. (A) Double immunohistochemistry for Tie-2 (red) and a smooth muscle actin (aSMA) (blue). Tie-2 was co-stained with aSMA, suggesting Tie-2 expression in pericytes surrounding capillaries. (B) Double staining with Tie-2 (red) and CD34 (blue) antibodies. Colocalisation of Tie-2 with CD34 was found only in capillaries with small lumen (red arrowheads) but not in tiny vessels lacking luminal structures (black arrowheads), suggesting the absence of Tie-2 expression at the initial stage of angiogenesis. (C) Tie-2 expression in capillary endothelium. Expression of Tie-2 and CD34 in a section from patient No 6 was shown in the three photomicrographs as follows. Left panel: Tie-2 immunoreactivity before the addition of CD34 antibody; middle panel: double staining for Tie-2 and CD34; right panel: CD34 expression after removal of Tie-2 staining by immersing in ethanol. (D) Double staining for Tie-2 (red) and proliferating cell nuclear antigen (PNCA) (blue). (E) Single staining for CD34 in a section adjacent to (D). PCNA was frequently overexpressed in Tie-2 positive endothelial and pericytic cells as indicated by black arrowheads. Inset in $(D)$ is a representative capillary showing both immunoreactivities. Note several CD34 positive cells in (E) are negative for Tie-2 and PCNA in (D) (red arrowheads). Asterisks in (D) and (E) represent large vessels which did not react with either Tie-2 or PCNA or CD34. Bars in each panel = $50 \mu \mathrm{m}$. 
A \begin{tabular}{l|llllllllll} 
Case: & 1 & 2 & 3 & 4 & 5 & 6 & 7 & 8 & 9 & 10 \\
Tie-1 - & & & & & & & & & & \\
\end{tabular} Tie-2

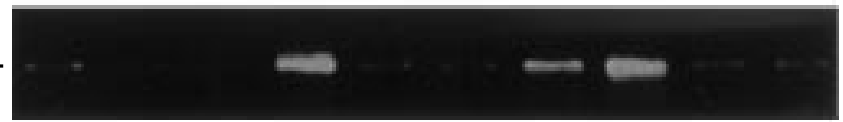

G3PDH

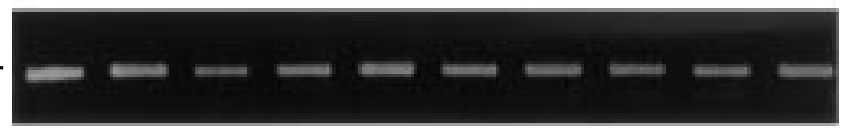

B

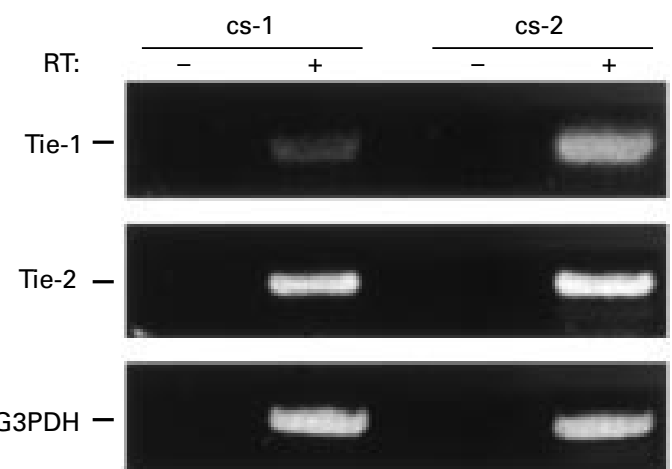

Figure 4 Reverse transcriptase polymerase chain reaction (RT-PCR) of Tie-1 and Tie-2. (A) Tie-1 and Tie-2 in synovial tissues. Total RNA was extracted from each synovium and cDNA was synthesised from $1 \mu \mathrm{g} R N A$. PCR was performed with specific primers corresponding to each cDNA. PCR cycles to amplify Tie-1, Tie-2, and G3PDH were 35, 35, and 25 cycles, respectively. Amplified PCR products were then electrophoresed onto $1.5 \%$ agarose gel. To rule out possible genomic DNA contamination, control experiments were also performed without $R T$ using the same reactions, and showed no amplification (data not shown). (B) Tie-1 and Tie-2 in cultured synoviocytes. Cultured synoviocytes obtained from two independent patients with $R A$ (cs-1 and cs-2) and subcultured five times were subjected to RT-PCR with the same conditions as described above.

RELATION BETWEEN TIE-1 AND TIE-2 EXPRESSION AND ANGIOGENESIS

Expression of Tie-1 and Tie-2 was also seen in vascular walls of all RA synovia. In contrast with synovial lining and stromal cells, however, there was no significant difference in the expression profile between Tie- 1 and Tie- 2 in the vascular wall. Although there was a regional variability, and no obvious correlation between Tie receptor expression and clinical features, Tie receptors tended to express intensely in capillaries located underneath the papillary proliferated synovium (fig 1A). Double staining with antibody against aSMA, which is expressed in pericytes as well as smooth muscle cells, showed that Tie-1 and Tie-2 were expressed in pericytes surrounding capillaries (fig 3A). Co-staining with CD34, a marker for endothelial cells, confirmed the expression of Tie receptors in the endothelium, though the expression of CD34 was limited to the inner surface of the endothelium (figs $3 \mathrm{~B}$ and $\mathrm{C}$ ). In addition to the small vessels which were stained by both CD34 and Tie receptors, many luminal structures composed of only CD34 stained cells could be seen among capillaries scattered over active synovia (fig 3B).
In the next step we examined whether expression of Tie receptors in the vasculature in RA synovium was associated with cell proliferation, and if so, we then determined whether Tie positive vessels were newly synthesised or existing blood vessels. Double staining with Tie-2 and PCNA and single staining with CD34 were performed using adjacent tissue sections. Overexpression of PCNA was often seen in the endothelium and surrounding pericytes of Tie-2 positive capillaries (fig 3D). Most vessels were stained by both Tie- 2 and PCNA. However, the vascular wall of a small proportion of vessels did not stain with either PCNA or Tie-2, even though CD34 was definitely expressed in the endothelium (figs $3 \mathrm{D}$ and $\mathrm{E})$.

RT-PCR OF TIE-1 AND TIE-2

To confirm and compare the expression levels of Tie- 1 and Tie- 2 in each tissue sample, RT-PCR was performed using specific primers to total RNA extracted from synovial tissues. Despite the use of equal volumes of RT products, there was some variability in the expression levels of G3PDH, which was used as internal control (fig 4A). However, specific PCR products of Tie-1 and Tie-2 were noted in almost all tissue samples, and the amount of PCR product reflected the expression levels seen in immunohistochemistry. Tie- 1 and Tie- 2 were amplified to maximum levels in patient No 4, who showed the highest expression of these proteins in immunohistochemistry.

To examine whether synoviocytes express Tie receptors in vitro, RT-PCR was performed on cultured fibroblastic synoviocytes derived from two independent patients with RA. As with synovial tissues, both Tie-1 and Tie-2 were amplified from cDNA of cultured synoviocytes without notable difference (fig 4B).

\section{Discussion}

Although Tie-1 and Tie-2 protein tyrosine kinase receptors have been considered to be expressed only in the endothelium of actively growing vessels, there is sufficient evidence at present indicating that these receptors are also expressed in quiescent endothelium or in other types of cells, including circulating haematopoietic progenitor cells. ${ }^{19}{ }^{20}$ We extended these studies by demonstrating in the present work that Tie receptors are also widely expressed in the pathologically abnormal synovial tissue of RA, including synovial lining cells, stromal cells, pericytes, and endothelial cells. We also demonstrated the expression of Tie receptors in cultured synoviocytes. In normal synovial tissues, however, these Tie receptors were limited to endothelial cells of small vessels.

In our sample group of patients with $\mathrm{RA}$, Tie- 1 and Tie- 2 were expressed in all RA synovia, and intense expression was seen in actively proliferated regions. Furthermore, our results showed that the distribution of Tie-1 expressing cells was different from that of cells positive for Tie-2 in synovial lining and stromal cells. As summarised in table 4, Tie-1 was expressed in the full thickness of the papillary proliferated synovial lining cells, whereas Tie-2 
Table 4 Summary of Tie-1 and Tie-2 expression in synovial tissues

\begin{tabular}{|c|c|c|c|c|}
\hline & \multicolumn{2}{|l|}{ Synovial lining cells } & \multicolumn{2}{|l|}{ Stromal cells } \\
\hline & CD68+/vimentin- & CD68-/vimentin+ & CD68+/vimentin- & CD68-/vimentin + \\
\hline Tie-1 & + & + & + & + \\
\hline Tie-2 & - & ++ & - & ++ \\
\hline PCNA* & - & ++ & $\mathrm{ND}^{\star}$ & ND \\
\hline
\end{tabular}

$\star \mathrm{PCNA}=$ proliferating cell nuclear antigen; $\mathrm{ND}=$ not determined.

expression was restricted to fibroblast derived cells (CD68-/vimentin+) located in the basal layer. Similar to the synovial lining cells, Tie-2 expression in stromal cells was limited to type A fibroblastic cells (CD68-/vimentin+) but not to type $B$ macrophage-derived cells (CD68+/vimentin-), whereas Tie-1 expression did not depend on the phenotype. Our results also showed the preferential expression of PCNA in cells of the basal layer. These results are consistent with previous findings that basal layer cells of synovial lining display fibroblastic phenotype and retain a proliferative capacity, whereas the superficial cells display a phenotype of differentiated macrophages. ${ }^{21}$

The exact functional role of these Tie receptors in RA synovium and the expression of angiopoietin-1 and/or -2 ligands for Tie-2 receptor in synovial tissue, remain unknown at present. However, the intense expression of these Tie receptors in the inflamed synovium suggests that Tie- 1 and Tie- 2 play some part in the pathophysiology of RA synovitis. Furthermore, the restricted expression of Tie- 2 in CD68-/vimentin+ cells suggests some specific functional role for Tie-2 in the differentiation of these cell from fibroblastic phenotype. Moreover, overexpression of PCNA in Tie-2 positive basal layered cells may be indicative of the direct involvement of these cells in the proliferation of lining cells.

In vasculature tissue of the RA synovium, our results showed the expression of Tie receptors in the endothelium and surrounding pericytes of proliferating capillaries located underneath the papillary stratified lining cells, without obvious differences in the expression patterns of Tie- 1 and Tie-2. In addition, overexpression of PCNA was restricted to Tie positive vessels. These results suggest the involvement of Tie receptors in vascularisation of the RA synovium, as well as the possible function of Tie-1 and Tie-2 as angiogenic factors under pathophysiological conditions. Previous studies have shown that in several pathological conditions, including malignant tumours, the expression of Tie receptors correlates well with the degree of angiogenesis. ${ }^{22}{ }^{23}$ These findings, together with our results for the expression profiles in synovial lining and stromal cells, suggest that the Tie-angiopoietin system plays a part in angiogenesis, synovial proliferation, and remodelling of RA pannus. Further experiments, including studies to examine the biological function of Tie- 1 and Tie-2 and immunohistochemistry for angiopoietins, are necessary for a better understanding of the pathophysiology of RA synovitis.
The origin and differentiation of vascular cells within the inflamed synovium are not well understood. However, clearly, based on the serial analysis of surface antigens, it seems that many endogenous haematopoietic cell precursors are present in synovial tissue of RA. ${ }^{24}$ Among these, a high density of CD34 positive cells have been shown in peripheral blood and in synovial tissue. ${ }^{25}$ In addition to serving as a marker for mature endothelium, CD34 is also expressed during early stages of angiogenesis. ${ }^{2627}$ Furthermore, it seems that CD34 positive cells in peripheral blood still retain a proliferative capacity as progenitor cells to both lymphocytes and endothelial cells. ${ }^{28}{ }^{29}$ Interestingly, we found many luminal structures surrounded by CD34 positive cells within capillary-rich areas, which might be indicative of immature capillary endothelium. Although a lineage between CD34 and Tie receptors, which has already been shown in lymphopoietic cells, ${ }^{30}$ has not yet been reported in the angiogenic process, it is possible that CD34 positive endothelial progenitor cells preexisting in, or infiltrating, synovial tissue from peripheral blood, differentiate into endothelial cells. Subsequent processes might include the induction of Tie receptors in the endothelium itself or in the surrounding undifferentiated cells for further growth of the vessels, in cooperation with the secreted angiopoietins. Elucidation of the mechanism underlying Tie receptor induced angiogenesis may allow the design of new treatments for RA.

In summary, we have shown here that Tie-1 and Tie-2 tyrosine kinase receptors are widely expressed in the proliferating synovium of RA, including synovial lining and stromal cells as well as the vascular system. We also showed that such expression is dependent on the proliferative activity of the RA synovium. Our results suggest the functional importance of Tie receptors in synovial proliferation and angiogenesis in RA synovitis.

We thank Mrs Motoki, Mrs Fukahori, and Mr Kawata for the preparation of histological sections, and Dr Kohno and Dr preparation of histological sections, and Dr Kohno and Dr
Kawabe for providing tissue samples and cultured synoviocytes, Kawabe for
respectively.

1 Stevens CR, Blake DR, Merry P, Revell PA, Levick JR. A comparative study by morphometry of the microvasculature in normal and rheumatoid synovium. Arthritis Rheum 1991;34:1508-13.

2 Walsh DA, Wade M, Mapp PI, Blake DR. Focally regulated endothelial proliferation and cell death in human synovium. Am J Pathol 1998;152:691-702.

3 Cronstein BN, Weissmann G. The adhesion molecules of Cronstein BN, Weissmann G. The adhesion mo
inflammation. Arthritis Rheum 1993;36:147-57.

4 Koch AE. Angiogenesis: implications for rheumatoid arthriKoch AE. Angiogenesis: implications for
tis. Arthritis Rheum 1998;41:951-62.

5 Firestein GS. Starving the synovium: angiogenesis and inflammation in rheumatoid arthritis. J Clin Invest 1999;103:3-4

6 Arsenault AL, Lhotak S, Hunter WL, Banquerigo ML, Brahn E. Taxol involution of collagen-induced arthritis: ultrastructural correlation with the inhibition of synovitis and neovascularization. Clin Immunol Immunopathol 1998;86:280-9.

7 Storgard CM, Stupack DG, Jonczyk A, Goodman SL, Fox RI, Cheresh DA. Decreased angiogenesis and arthritic disease in rabbits treated with an alpha v beta3 antagonist. J Clin Invest 1999;103:47-54.

8 Schnurch H, Risau W. Expression of tie-2, a member of a novel family of receptor tyrosine kinases, in the endothelial

9 Sato TN, Qin Y, Kozak CA, Audus KL. Tie-1 and tie-2 define another class of putative receptor tyrosine kinase genes expressed in early embryonic vascular system. Proc Natl Acad Sci USA 1993;90:9355-8. 
10 Sato TN, Tozawa Y, Deutsch U, Wolburg BK, Fujiwara Y, Gendron MM, et al. Distinct roles of the receptor tyrosine kinases Tie-1 and Tie-2 in blood vessel formation. Nature 1995;376:70-4

11 Dumont DJ, Gradwohl G, Fong GH, Puri MC, Gertsenstein $\mathrm{M}$, Auerbach $\mathrm{A}$, et al. Dominant-negative and targeted null mutations in the endothelial receptor tyrosine kinase, tek, reveal a critical role in vasculogenesis of the embryo. Genes Dev 1994;8:1897-909.

12 Davis S, Aldrich TH, Jones PF, Acheson A, Compton DL, Jain V, et al. Isolation of angiopoietin-1, a ligand for the TIE2 receptor, by secretion-trap expression cloning. Cell 1996;87:1161-9.

13 Maisonpierre PC, Suri C, Jones PF, Bartunkova S, Wiegand SJ, Radziejewski C, et al. Angiopoietin-2, a natural antagonist for Tie2 that disrupts in vivo angiogenesis. Science 1997;277:55-60.

14 Suri C, Jones PF, Patan S, Bartunkova S, Maisonpierre PC, Davis S, et al. Requisite role of angiopoietin-1, a ligand for the TIE2 receptor, during embryonic angiogenesis. Cell 1996;87:1171-80.

15 Asahara T, Chen D, Takahashi T, Fujikawa K, Kearney M, Magner $M$, et al. Tie 2 receptor ligands, angiopoietin-1 and angiopoietin-2, modulate VEGF- induced postnatal neovascularization. Circ Res 1998;83:233-40.

16 Arnett FC, Edworthy SM, Bloch DA, McShane DJ, Fries JF, Cooper NS, et al. The American Rheumatism Association 1987 revised criteria for the classification of rheumatoid arthritis. Arthritis Rheum 1988;31:315-24.

17 Sugiyama M, Tsukazaki T, Yonekura A, Matsuzaki S, Yamashita S, Iwasaki K. Localisation of apoptosis and expression of apoptosis related proteins in the synovium of patients with rheumatoid arthritis. Ann Rheum Dis 1996;55:442-9.

18 Nakashima M, Ito M, Ohtsuru A, Alipov GK, Matsuzaki S, Nakayama $\mathrm{T}$, et al. Expression of parathyroid hormone (PTH)-related peptide (PTHrP) and PTH/PTHrP receptor in giant cell tumour of tendon sheath. J Pathol tor in giant cell

19 Wong AL, Haroon ZA, Werner S, Dewhirst MW, Greenberg CS, Peters KG. Tie2 expression and phosphorylation in angiogen
20 Yuan HT, Suri C, Yancopoulos GD, Woolf AS. Expression of angiopoietin-1, angiopoietin-2, and the Tie-2 receptor tyrosine kinase during mou

21 Enzinger FM, Weiss SW. Benign tumors and tumor-like lesions of synovial tissue. In: Enzinger FM, Weiss SW, eds. Soft tissue tumors. St Louis: Mosby, 1995:735-55.

22 Peters KG, Coogan A, Berry D, Marks J, Iglehart JD, Kontos CD, et al. Expression of Tie2/Tek in breast tumour vasculature provides a new marker for evaluation of tumour angiogenesis. Br J Cancer 1998;77:51-6.

23 Stratmann A, Risau W, Plate KH. Cell type-specific expression of angiopoietin-1 and angiopoietin-2 suggests a role in glioblastoma angiogenesis. Am J Pathol 1998;153:145966.

24 Athanasou NA, Quinn J. Immunocytochemical analysis of human synovial lining cells: phenotypic relation to other
marrow derived cells. Ann Rheum Dis 1991;50:311-15.

25 Santiago SF, Sullivan C, Rappa D, Carsons SE. Distinct alterations in lineage committed progenitor cells exist in the peripheral blood of patients with rheumatoid arthritis and primary Sjögren's syndrome. J Rheumatol 1996;23: $439-46$.

26 Wood HB, May G, Healy L, Enver T, Morriss KG. CD34 expression patterns during early mouse development are related to modes of blood vessel formation and reveal additional sites of hematopoiesis. Blood 1997;90:2300-11.

27 Hamaguchi I, Huang XL, Takakura N, Tada J, Yamaguchi $\mathrm{Y}$, Kodama $\mathrm{H}$, et al. In vitro hematopoietic and endothelial cell development from cells expressing TEK receptor in murine aorta-gonad-mesonephros region. Blood 1999;93: 1549-56.

28 Asahara T, Murohara T, Sullivan A, Silver M, van der Zee $\mathrm{R}, \mathrm{Li} \mathrm{T}$, et al. Isolation of putative progenitor endothelial cells for angiogenesis. Science 1997;275:964-7.

29 Hirashima M, Kataoka H, Nishikawa S, Matsuyoshi N, Nishikawa S. Maturation of embryonic stem cells into endothelial cells in an in vitro model of vasculogenesis. Blood 1999;93:1253-63.

30 Yano M, Iwama A, Nishio H, Suda J, Takada G, Suda T. Expression and function of murine receptor tyrosine
kinases, TIE and TEK, in hematopoietic stem cells. Blood 1997;89:4317-26. 